\title{
Stability of Relativistic Matter With Magnetic Fields
}

\author{
Elliott H. Lieb ${ }^{1}$, Heinz Siedentop ${ }^{2}$, and Jan Philip Solovej ${ }^{3}$ \\ ${ }^{1}$ Department of Physics, Jadwin Hall, Princeton University, P. O. Box 708, Princeton, New Jersey 08544 \\ ${ }^{2}$ Mathematik, Universität Regensburg, D-93040 Regensburg, Germany \\ ${ }^{3}$ Department of Mathematics, Aarhus University, DK-8000 Aarhus C, Denmark
}

\begin{abstract}
Stability of matter with Coulomb forces has been proved for non-relativistic dynamics, including arbitrarily large magnetic fields, and for relativistic dynamics without magnetic fields. In both cases stability requires that the fine structure constant $\alpha$ be not too large. It was unclear what would happen for both relativistic dynamics and magnetic fields, or even how to formulate the problem clearly. We show that the use of the Dirac operator allows both effects, provided the filled negative energy 'sea' is defined properly. The use of the free Dirac operator to define the negative levels leads to catastrophe for any $\alpha$, but the use of the Dirac operator with magnetic field leads to stability.
\end{abstract}

PACS numbers: 03.65.-w, 11.10.-z, 12.20.-m

Since the work of Dyson and Lenard in 1967 [1] it has been well understood that matter consisting of $N$ electrons and $K$ static (but arbitrarily positioned), point nuclei, and governed by nonrelativistic quantum mechanics, is stable. (See also [2] and see [3], [1] for reviews.) This means that the ground state energy is finite and is bounded below by a universal constant times the number of particles, i.e., $E_{0} \geq$ const. $(N+K)$. If the nuclei are either non-static or non-point the problem becomes easier. The reason the nuclei are taken to be static is that they are so massive compared to the electron that if the nuclear mass (or the nuclear radius of $10^{-13} \mathrm{~cm}$, for that matter) played any important role then ordinary matter would have to look very different from what it does; electron orbital radii would be many orders of magnitude smaller than the Bohr radius.

We know that atoms, molecules and bulk matter exist and have ground states that are well described by the Schrödinger equation with infinitely massive, point nuclei. This description needs corrections, however, even at the low energies appropriate to atomic structure. Effects due to special relativity and interactions of electrons with (quantized or unquantized) magnetic fields are significant. Usually, one appeals to quantum electrodynamics (QED) for these corrections, but that theory, as usually formulated, is not known to exist outside of perturbation theory. More importantly, the effects mentioned are often not perturbative, despite the fact that the fine structure constant $\alpha=e^{2} / \hbar c \sim 1 / 137$ is small. It is well known, for instance that the Dirac equation breaks down when $Z \alpha(Z=$ nuclear charge), the quantity of greatest importance, is 1 . The quantity $Z \alpha$ is not at all small for Uranium and hence relativistic and electrodynamic ef- fects are not small. It is sometimes said that some proper high energy theory (e.g., QCD or strings) will straighten things out, but it is difficult to believe that we cannot have a theory of chemistry without theoretical insertions in the $\mathrm{GeV}$ range.

With this in mind, attempts have been made over the years to approach a theory of matter by ever increasing generalizations of the many-body Schrödinger equation, all the while maintaining the many-body aspect and a non-perturbative, rigorous treatment. One significant step was the introduction of a 'relativistic' kinetic energy into the Schrödinger equation by replacing $p^{2} / 2 m$ by $\sqrt{p^{2}+m^{2}}-m c^{2}$ (with $\mathbf{p}=-i \hbar \nabla$ as usual) or, more simply, by $p=|\mathbf{p}|$. We can also include a (non-quantized) magnetic field $\mathbf{B}(\mathbf{x})=\operatorname{curl} \mathbf{A}(\mathbf{x})$ acting on the orbital motion, i.e., we use $|\mathbf{p}+\mathbf{A}(\mathbf{x})|$. The Hamiltonian (in appropriate units, in which all dimensional parameters reside in $\alpha$ and $p=-i \nabla)$ is then

$$
H_{\mathrm{rel}} \equiv \sum_{i=1}^{N}\left|\mathbf{p}_{i}+\mathbf{A}\left(\mathbf{x}_{i}\right)\right|+\alpha V_{\mathrm{c}},
$$

where the Coulomb potential of the electrons (with coordinates $\mathbf{x}_{i}$ ) and nuclei (with coordinates $\mathbf{R}_{j}$ ) is

$$
\begin{aligned}
V_{\mathrm{c}}= & -Z \sum_{i=1}^{N} \sum_{j=1}^{K}\left|\mathbf{x}_{i}-\mathbf{R}_{j}\right|^{-1}+\sum_{1 \leq i<j \leq N}\left|\mathbf{x}_{i}-\mathbf{x}_{j}\right|^{-1} \\
& +Z^{2} \sum_{1 \leq i<j \leq K}\left|\mathbf{R}_{i}-\mathbf{R}_{j}\right|^{-1} .
\end{aligned}
$$

(It is purely for convenience that we take the nuclei to have the same $Z$.)

For $A \equiv 0$, Conlon [5] showed that matter was stable in this theory if $\alpha$ is 'small enough'. The constants were improved in [6] and finally in [7] stability was proved all the way up to the critical value appropriate to a 'hydrogenic atom' (which is $Z \alpha \leq 2 / \pi$ in this theory [8], [9] instead of $Z \alpha \leq 1$ as in the Dirac theory) if and only if $\alpha$ itself is less than some critical value $\alpha_{c}$ (independent of $Z$ ). In [7] it was shown that $\alpha_{c}>1 / 94$.

For arbitrary, nonzero $A$ stability was shown to hold for (1) in [10] if $\alpha<\tilde{\alpha}$, where

$$
1 / \tilde{\alpha} \equiv\left(\frac{\pi}{2}\right) Z+2.80 Z^{2 / 3}+1.30,
$$

which permits $Z \leq 59$ for $\alpha=1 / 137$. Eq. (2) will be useful later. 
Another important ingredient was the inclusion of arbitrarily large magnetic fields and, at the same time the replacement of $p^{2} / 2 m$ by the non-relativistic Pauli operator (in suitable units)

$$
\mathcal{T}_{\mathbf{A}} \equiv[\boldsymbol{\sigma} \cdot(\mathbf{p}+\mathbf{A}(\mathbf{x}))]^{2}=(\mathbf{p}+\mathbf{A}(\mathbf{x}))^{2}+\boldsymbol{\sigma} \cdot \mathbf{B} .
$$

This field, $\mathbf{A}(\mathbf{x})$, can be viewed in two ways: either as an applied external field or as some sort of average (in the sense of Hartree theory) of the field produced by all the electrons themselves. The replacement of $p^{2}$ by $\left[(\mathbf{p}+\mathbf{A}(\mathbf{x})]^{2}\right.$ is a generalization that has long been known to cause no problems with stability in the non-relativistic case. The essential new ingredient in the Pauli operator is the Zeeman term $\boldsymbol{\sigma} \cdot \mathbf{B}$, which results in the fact that there is no lower bound to the energy, even for hydrogen [11]. (We have in mind here that we make no restriction on the field $\mathbf{A}$, which is allowed to be arbitrarily large.) To remedy the instability due to arbitrarily large fields we add the field energy

$$
H_{\text {field }} \equiv[8 \pi \alpha]^{-1} \int B(\mathbf{x})^{2} d^{3} x
$$

in the units we have been employing.

For a single atom it was shown a decade ago that the addition of $H_{\text {field }}$ stabilizes the system ( $E_{0}$ is finite) if and only if $Z \alpha^{2}$ is less than some comfortably large, but finite value [12]. The 'only if' part relies on a deep result in 13 .

Stability with arbitrary $N$ and $K$ and kinetic energy (3) was first solved by Fefferman (unpublished) for sufficiently small $\alpha$ and $Z$; see [14] for an announcement. Shortly thereafter a simple proof with good constants (e.g., we can take $Z=1050$ when $\alpha=1 / 137$ ) was given [15] but, as in the 'relativistic case' mentioned above there is an $\alpha_{c}$ such that the system is unstable whenever $\alpha>\alpha_{c}$, no matter how small Z may be. Using the developments in [15], it was shown in [16] that this nonrelativistic theory remains stable when the magnetic field is quantized, provided an ultraviolet cutoff is introduced.

Thus, two essential modifications of the Schrödinger energy (replacing $p^{2}$ by $p$ or the inclusion of spinmagnetic field interaction) require a bound on $\alpha$, in addition to a bound on $Z \alpha$ or $Z \alpha^{2}$, for many-body stability. One might surmise that the combination of the two would lead to further difficulties - even, possibly, instability for all $\alpha$. This is not so, as we show in this letter. Indeed, our result is a bit surprising for it reveals an unanticipated delicacy about the manner of defining an electron in Dirac's theory.

The best way to include relativity and spin-field interaction is to use the Dirac operator

$$
\mathcal{D}_{\mathbf{A}} \equiv \not \mathbf{p}+\mathbf{A}(\mathbf{x})+\beta m \text {. }
$$

As usual, $\boldsymbol{\phi} \equiv \boldsymbol{\alpha} \cdot \mathbf{O}$, where $\alpha_{i}$ and $\beta$ are the $4 \times 4$ Dirac matrices. Our many-body Hamiltonian (with no pair production) is then, formally,

$$
H_{\text {Dirac }} \equiv \sum_{i=1}^{N} \mathcal{D}_{\mathbf{A}}(i)+\alpha V_{\mathrm{c}}+H_{\text {field }}
$$

Unlike the usual (or the Pauli) kinetic energy operators, the Dirac operator is not bounded below, so we use Dirac's prescription of filling the negative energy 'sea'. Concretely, this means that all the electrons lie in the positive spectral subspace of the Dirac operator, i.e., if $\Lambda^{+}$denotes the projector onto the positive spectral subspace for all the electrons then we allow only many-body functions $\psi$ (which are antisymmetric functions of space and four component spinors) that satisfy

$$
\Lambda^{+} \psi=\psi
$$

Another way to say this is that the ground state energy is given by the infimum of the variational quantity (with respect to $\psi$ and $A(\mathbf{x})$ )

$$
\mathcal{E}(\psi) \equiv\left\langle\psi\left|\Lambda^{+} H_{\operatorname{Dirac}} \Lambda^{+}\right| \psi\right\rangle /\left\langle\Lambda^{+} \psi \mid \Lambda^{+} \psi\right\rangle .
$$

The idea of studying this problem goes back to Brown and Ravenhall [17], who studied a single Helium atom with $\mathbf{A}=\mathbf{0}$ and $\Lambda_{+}$being the positive spectral subspace for the free Dirac operator, $\mathcal{D}_{\mathbf{0}}$. This problem with a single general atom, i.e., $K=1$, but $N$ and $Z$ arbitrary, has recently been shown stable, if $Z \alpha \leq 2 /(\pi / 2+2 / \pi)$, and instable otherwise [18].

While the Hamiltonian $H$ does not depend on the definition of the positive spectral subspace, and it always includes the $\mathbf{A}$ field, the meaning of $\Lambda^{+}$, and hence $E_{0}$, does depend on the subspace.

There's the rub! Which Dirac operator should we use to define $\Lambda^{+}$? There are at least two significant choices. One is the free Dirac operator $\mathcal{D}_{\mathbf{0}}=\not \mathbf{p}+\beta m$, which is the usual choice. The other is the Dirac operator with the magnetic field $\mathcal{D}_{\mathbf{A}}$. The latter seems more important since an electron can never get rid of its own magnetic field. Moreover, the choice $\mathcal{D}_{\mathbf{0}}$, is not gauge invariant, i.e., the multiplication of an electron wave function by a spatially varying phase usually takes a positive energy function into a mixture of positive and negative energy functions. The second choice is manifestly gauge invariant.

This issue is usually not clearly stated in field theory textbooks, but whatever one might think about the appropriateness of either definition the interesting and surprising fact is that "stability" of matter can settle the argument. The following two theorems are the main results in [19]. They show that one choice is valid and the other is not.

THEOREM 1 (Stability with the magnetic Dirac operator): 
If the electron wave function is restricted to lie in the positive spectral subspace of the Dirac operator $\mathcal{D}_{\mathbf{A}}$ then $H_{\text {Dirac }}$ is stable (i.e., $H_{\text {Dirac }}>0$ ) provided $\alpha$ and $Z$ are small enough. In particular, if we define $\alpha_{c}$ to be the

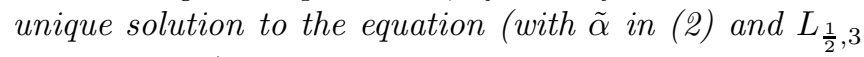
defined below)

$$
1-\left(\alpha_{c} / \tilde{\alpha}\right)^{2}=\left(16 \pi L_{\frac{1}{2}, 3} \alpha_{c}\right)^{2 / 3}
$$

then $\alpha \leq \alpha_{c}$ suffices for stability. In particular, $Z=56$ and $\alpha=1 / 137$, or $Z=1$ and $\alpha=1 / 9$ are sufficient for stability.

We shall outline the proof of this theorem here; details can be found in 19 .

THEOREM 2 (Instability with the free Dirac operator): If the electron wave function is restricted to lie in the positive spectral subspace of the free Dirac operator $\mathcal{D}_{0}$ then the Hamiltonian $H_{\text {Dirac }}$ is unstable. More precisely, for any given values of $\alpha>0$ and $Z>0$, there are (sufficiently large) particle numbers $N$ and $K$ for which the infimum of $\mathcal{E}(\psi)$ is $-\infty$.

Remark on the proof of Theorem 2: This requires a complicated construction of variational functions with arbitrarily negative energy. It is necessary to construct $N$ particle antisymmetric trial functions, $\psi$, of space-spin and also corresponding magnetic potentials $\mathbf{A}(\mathbf{x})$. See [19].

Proof of Theorem 1: Using (11) and (2) we have that

$$
V_{\mathrm{c}} \geq-\frac{1}{\tilde{\alpha}} \sum_{i=1}^{N}\left|\mathbf{p}_{i}+\mathbf{A}\left(\mathbf{x}_{i}\right)\right| .
$$

and hence $\mathcal{E}(\psi)-H_{\text {field }}$ is bounded below by the sum of the negative eigenvalues of the one-body operator

$$
h:=\Lambda^{+}\left(\mathcal{D}_{\mathbf{A}}-\kappa|\mathbf{p}+\mathbf{A}(\mathbf{x})|\right) \Lambda^{+},
$$

with $\kappa \equiv \alpha / \tilde{\alpha}$. I.e., $\mathcal{E}(\psi)-H_{\text {field }} \geq-\operatorname{Tr} h_{-}$, where $h_{-} \equiv$ $[|h|-h] / 2$.

Next we note the BKS inequality [20]: If $X>0, Y>0$ are self-adjoint operators, then

$$
\operatorname{Tr}[X-Y]_{-} \leq \operatorname{Tr}\left[X^{2}-Y^{2}\right]_{-}^{1 / 2} .
$$

We use (平) with $X=\Lambda^{+} \mathcal{D}_{\mathbf{A}} \Lambda^{+}$and $Y=\Lambda^{+} \kappa \mid \mathbf{p}+$ $\mathbf{A}(\mathbf{x}) \mid \Lambda^{+}$. But we note that $\mathcal{D}_{\mathbf{A}}^{2}=\mathcal{T}_{\mathbf{A}}+m^{2}>\mathcal{T}_{\mathbf{A}} \geq(\mathbf{p}+$ $\mathbf{A})^{2}-B(\mathbf{x})$ and we note that $X^{2}=\Lambda^{+} \mathcal{D}_{\mathbf{A}}^{2} \Lambda^{+}\left(\right.$since $\mathcal{D}_{\mathbf{A}}$ commutes with $\left.\Lambda^{+}\right)$, while $Y^{2} \leq \kappa^{2} \Lambda^{+}(\mathbf{p}+\mathbf{A}(\mathbf{x}))^{2} \Lambda^{+}$. Thus we conclude that for every choice of $\mathbf{A}$ and $\psi$

$$
\begin{aligned}
\mathcal{E}(\psi)-H_{\text {field }} & \geq-\operatorname{Tr}\left[\left(1-\kappa^{2}\right)(\mathbf{p}+\mathbf{A}(\mathbf{x}))^{2}-B(\mathbf{x})\right]_{-}^{1 / 2} \\
& \geq-4\left(1-\kappa^{2}\right)^{-3 / 2} L_{\frac{1}{2}, 3} \int B(\mathbf{x})^{2} d^{3} x
\end{aligned}
$$

by the Lieb-Thirring inequality for the sum of the square roots of the negative eigenvalues of a Schrödinger operator $(\mathbf{p}+\mathbf{A}(\mathbf{x}))^{2}+V(\mathbf{x})$ with arbitrary $\mathbf{A}$ and arbitrary potential $V$. This is

$$
\operatorname{Tr}\left[(\mathbf{p}+\mathbf{A}(\mathbf{x}))^{2}+V(\mathbf{x})\right]_{-}^{1 / 2} \leq L_{\frac{1}{2}, 3} \int V_{-}^{2}(\mathbf{x}) d^{3} x .
$$

Note that in (5) there is a factor of 4 on the right side because the trace includes a trace over 4-dimensional spinors; in (6) there is no such trace, i.e., (5) is for spinless particles. It is known 21] that $L_{\frac{1}{2}, 3}<0.06003$.

The factor 4 in (5) can be reduced to 2 , as one might expect physically; details are in [19]. Theorem 1 then follows easily from (5).

The authors thank the following organizations for their support: the Danish Science Foundation, the European Union, TMR grant FMRX-CT 96-0001, the U.S. National Science Foundation, grant PHY95-13072A01, and NATO, grant CRG96011

[1] F.J. Dyson and A. Lenard, J. Math. Phys. 8, 423 (1967); 9, 698 (1967).

[2] E.H. Lieb and W.E. Thirring, Phys. Rev. Lett. 35, 687 (1975); Errata 35, 1116 (1975).

[3] E.H. Lieb, Rev. Mod. Phys. 48, 553 (1976).

[4] E.H. Lieb, Bull. Amer. Math. Soc. 22, 1 (1990).

[5] J.G. Conlon, Commun. Math. Phys. 94, 439 (1984).

[6] C. Fefferman and R. de la Llave, Rev. Math. Iberoamericana 2, 119 (1986).

[7] E.H. Lieb and H-T. Yau, Commun. Math. Phys. 118, 177 (1988).

[8] I. Herbst, Commun. Math. Phys. 53, 285 (1977).

[9] T. Kato, Perturbation Theory for Linear Operators in Grundl. der mathem. Wissen., 132, Springer (1966).

[10] E.H. Lieb, M. Loss, and H. Siedentop, Helv. Phys. Acta, 69, 974 (1996).

[11] J. Avron, I. Herbst, and B. Simon, Commun. Math. Phys. 79, 529 (1981).

[12] J. Fröhlich, E.H. Lieb and M. Loss, Commun. Math. Phys. 104, 251 (1986); E.H. Lieb and M. Loss, Commun. Math. Phys. 104, 271 (1986).

[13] M. Loss and H-T. Yau, Commun. Math. Phys. 104, 283 (1986).

[14] C. Fefferman, Proc. Natl. Acad. Sci. USA, 92, 5006 (1995).

[15] E.H. Lieb, M. Loss, and J.P. Solovej, Phys. Rev. Lett., 75, 985 (1995).

[16] L. Bugliaro, J. Fröhlich, and G.M. Graf, Phys. Rev. Lett. 77, 3494 (1996).

[17] G.E. Brown and D.G. Ravenhall, Proc. Roy. Soc. London A208, 552 (1952).

[18] D. Evans, P. Perry, and H. Siedentop, Commun. Math. Phys. 178, 733 (1996). 
[19] E.H. Lieb, H. Siedentop, and J.P. Solovej, Stability and Instability of Relativistic Electrons in Classical Electromagnetic fields, Jour. Stat. Phys., (accepted for publication). Preprint available at http://xxx.lanl.gov/abs/cond-mat/9610195.

[20] M.S. Birman, L.S. Koplienko, and M.Z. Solomyak, Sov. Math. 19, 1 (1975). Translation of Izvestija vyssich.

[21] E.H. Lieb, Commun. Math. Phys. 92, 473 (1984). 